298 cases. Organ-specific classification of triggered autoimmune diseases included cutaneous $(n=7140)$, digestive $(n=2323)$, endocrine $(n=1701)$, pulmonary $(n=1683)$, systemic/rheumatic $(n=1230)$, neurological $(n=1172)$, ocular $(n=349)$, cardiac $(n=322)$, haematological $(n=106)$ and renal $(n=96)$ diseases. The most frequently reported induced individual autoimmune diseases were psoriasis $(n=6401)$, enterocolitis $(n=1947)$, interstitial lung diseases $(n=1680)$, thyroiditis $(n=1076)$, hypophysitis $(n=492)$, demyelinating CNS diseases $(n=452)$, peripheral neuropathies $(n=431)$, lupus $(n=371)$, vasculitis $(n=339)$, uveitis $(n=298)$, vitiligo $(n=256)$ and sarcoidosis $(n=185)$.

Conclusions: The number and diversity of induced autoimmune disorders is increasing exponentially in parallel with the increased use of biological therapies in neoplasic diseases, which are currently representing $40 \%$ of reported cases. Management of these biologic-induced autoimmune diseases will be an increasing multidisciplinary clinical challenge in the daily practice in the next years.

Disclosure of Interest: None declared

DOI: 10.1136/annrheumdis-2018-eular.4257

\section{THU0666 COSTS ASSOCIATED WITH SWITCHING SUBCUTANEOUS TUMOUR NECROSIS FACTOR-A INHIBITOR IN THE TREATMENT OF IMMUNE-MEDIATED RHEUMATIC DISEASE}

J. Dalén' ${ }^{1}$, A. Svedbom ${ }^{1}$, K. Luttropp ${ }^{1}$, S. Kachroo ${ }^{2} .{ }^{1}$ Mapi, Stockholm, Sweden; ${ }^{2}$ Center for Observational and Real-World Evidence (CORE), Merck and Co., Inc., Kenilworth, NJ, USA

Background: Few studies have investigated the costs associated with switching subcutaneous tumour necrosis factor- $\alpha$ inhibitor (SC-TNFi) in the treatment of immune-mediated rheumatic disease (IMRD; rheumatoid arthritis, ankylosing spondylitis, and psoriatic arthritis), ${ }^{1-2}$ and to our knowledge no studies have been performed in a European setting.

Objectives: The objective of this retrospective observational study was to explore costs of health care resource utilisation (HCRU) associated with switching SCTNFi in patients with IMRD in Sweden.

Methods: Using data from Swedish Health Data Registers, adult, treatment naïve IMRD patients filling prescriptions between May 1st 2010 and December 31st 2015 for any of the currently available SC-TNFi (adalimumab, etanercept, certolizumab, and golimumab) were included. Prescriber specialty and department were used to exclude patients with SC-TNFi treatment unrelated to IMRD. A switch was defined as a filled prescription for a subsequent SC-TNFi within 60 days of first line discontinuation. HCRU, including non-DMARD medication, inpatient and specialised outpatient care, was captured 12 months pre- and post-treatment initiation. Eligible patients had at least 12 months follow-up, and were either persistent throughout or switched treatment during the follow-up period. The analysis was performed on a propensity score matched (PSM) cohort.

\section{Figure 1. Total Cost of Health Care Resource Utilization}

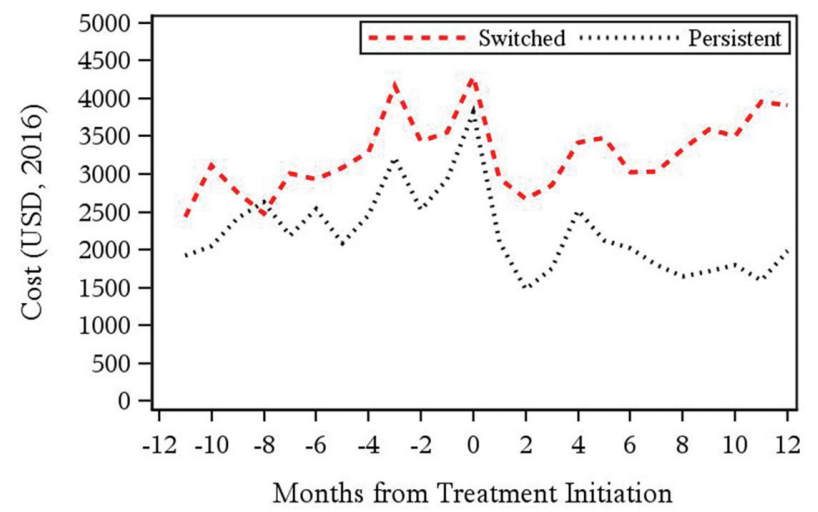

Abstract THU0666 - Figure 1 Total. cost of health care resource utilisation

Results: In total, 6213 eligible patients were identified, of which 5092 were persistent with their index therapy throughout the follow-up period while 1120 patients switched SC-TNFi. The PSM cohort was derived as "Persistent" vs. "Switched" with a total of 1120 matched pairs. Patients switching therapy had higher total
HCRU costs during the baseline period and maintained their average cost during the follow-up period (figure 1), whereas persistent patients decreased their average costs from the baseline period to the follow-up period. The average yearly cost during baseline and follow-up was USD 4,512 vs USD 4,690 for patients switching SC-TNFi, and USD 3,630 vs USD 2,662 for persistent patients. This cor responds to a difference in difference of USD 1,146 ( $p<0.001)$.

Conclusions: Similar to previous findings, ${ }^{3-4}$ patients switching maintained their higher costs, while persistent patients decreased their costs from baseline to the follow-up period. This further highlights the notion that persistence may not only serve as a marker for drug effectiveness, safety, and treatment satisfaction, but also for reduced HCRU and costs.

\section{REFERENCES :}

[1] Borah BJ, et al. Trends in RA patients' adherence to subcutaneous antiTNF therapies and costs. Curr Med Res Opin 2009;25(6):1365-77.

[2] Cannon GW, et al. Clinical outcomes and biologic costs of switching between tumor necrosis factor inhibitors in US veterans with rheumatoid arthritis. Adv Ther 2016;33(8):1347-59.

[3] Dalén J, et al. Treatment persistence among patients with immune-mediated rheumatic disease newly treated with subcutaneous TNF-alpha inhibitors and costs associated with non-persistence. Rheumatol Int 2016 Jul;36 (7):987-95.

[4] Dalén J, et al. Second-line treatment persistence and costs among patients with immune-mediated rheumatic diseases treated with subcutaneous TNF-alpha inhibitors. Rheumatol Int 2017;37(12):2049-58.

Disclosure of Interest: J. Dalén Employee of: Mapi, A. Svedbom Employee of: Mapi, K. Luttropp Employee of: Mapi, S. Kachroo Shareholder of: Merck and Co. Inc., Employee of: Merck and Co., Inc.

DOI: 10.1136/annrheumdis-2018-eular.6560

\section{THU0667 DISEASE, WORK AND PERSONAL RELATED FACTORS ASSOCIATED WITH PRESENTEEISM IN PATIENTS WITH RHEUMATOID ARTHRITIS: RESULTS FROM THE NATIONAL RHEUMATOID ARTHRITIS SOCIETY SURVEY (NRAS)}

S. Verstappen ${ }^{1}$, M. Gignac ${ }^{2}$, L. Lunt ${ }^{1}$, D. Beaton ${ }^{3}$, A. Bosworth ${ }^{4}$, M. Bezzant ${ }^{4}$ K. Walker-Bone ${ }^{5} .{ }^{1}$ CfMR, Manchester, UK; ${ }^{2}$ University of Toronto; ${ }^{3} \mathrm{IWH}$, Toronto, Canada; ${ }^{4}$ NRAS, Maidenhead; ${ }^{5}$ ARUK/MRC CfMHW, Southampton, UK

Background: At-work productivity loss (i.e. presenteeism) is a major problem for patients with rheumatoid arthritis (RA) and employers and could be a marker of long-term absenteeism. To develop interventions to prevent presenteeism, a better understanding of which factors are associated with presenteeism is needed. Objectives: To assess the association between disease and work related factors with presenteeism.

Methods: A large survey about employment amongst patients with RA was con ducted in the UK using an online platform and inviting NRAS members and nonmembers to participate. Patients completed the multi-item Workplace Activity Limitation Scale (WALS; range 0-36 worst score), a measure of presenteeism. Other job related questions included: occupation (NS-SEC coding), job demand questions, help from colleagues (categorised into: always/often, sometimes, rarely/ never) and a patient acceptable state questionnaire about work (PASS). Patients also completed the disease specific RAID questionnaire (score $0-10=$ worst score). Univariable and multivariable linear regression analyses were performed to assess the association between the disease and job related factors and presenteeism, adjusting for age and gender.

Results: 891 respondents were in paid work at the time of the survey $(51.5 \%$ working for others, $33.1 \%$ self-employed and $15.4 \%$ on temporary sick leave). The majority of participants were women (91.5\%) and $4.9 \%$ were aged $16-30$, $69.2 \% 31-54 \%$ and $25.8 \%$ aged $55-74$ years. Over half $(51.5 \%)$ had a higher managerial, administrative or professional occupations, $33.1 \%$ an intermediate occupation, and $15.4 \%$ a routine/manual occupation. Mean (SD) RAID score was 5.2 (2.2). $58.2 \%$ rated their current job performance somewhat/much worse than before the onset of their arthritis. In univariable regression analyses greater disease activity, less control, lower support from colleagues and being in a unacceptable disease state (PASS) were associated with higher levels of presenteeism. In multivariable analysis, disease activity, ability to influence work and a unaccept able disease state remained statistically significantly associated with presenteeism. 